\title{
La tomografía de tórax para tamizaje de COVID-19 en cirugía electiva de cáncer de cabeza y cuello. ¿Es suficiente?
}

\author{
Leonardo A. Barba-Valadez, José F. Gallegos-Hernández," Ariadna L. Benítez-Martínez y José A. \\ Ábrego-Vásquez \\ Hospital de Oncología, Centro Médico Nacional Siglo XXI, Instituto Mexicano del Seguro Social, Ciudad de México, México
}

\begin{abstract}
Resumen
Introducción: Los pacientes con cáncer de cabeza y cuello tienen alto riesgo de infección por SARS-CoV-2; la cirugía en ellos implica riesgo para pacientes, cirujanos, personal de salud, institución médica y sociedad, ya que se asocia a aerosolización prolongada e inadvertida y a procedimientos de urgencia que facilitan la ruptura de las medidas de protección del personal de salud. Objetivo: Conocer si los hallazgos tomográficos pulmonares son suficientes para identificar en forma preoperatoria a los pacientes con COVID-19. Métodos: Estudio retrospectivo, transversal y analítico de pacientes con neoplasias cervicofaciales candidatos a cirugía, evaluados preoperatoriamente mediante tomografía axial computarizada simple de tórax con base en la clasificación CO-RADS. En los pacientes CO-RADS $\geq 3$ se suspendió la cirugía y se realizó PCR por hisopado nasofaríngeo. Resultados: Se incluyeron 322 pacientes, todos sin síntomas de COVID-19. La tomografía fue positiva en 35 (10.87\%); en 30 se efectuó hisopado nasofaríngeo: 28 fueron negativos y dos, positivos; ninguno desarrolló síntomas de COVID-19. Conclusiones: La tomografía torácica no es útil como procedimiento único de tamizaje preoperatorio de COVID-19, ya que sus hallazgos son inespecíficos, con tasa alta de resultados falsos-positivos. La evaluación clínica, con PCR y tomografía es la mejor forma de pesquisa preoperatoria.
\end{abstract}

PALABRAS CLAVE: Cabeza y cuello. Cáncer. COVID-19. Pesquisa. PCR. Tomografía.

\section{Chest tomography for COVID-19 screening in head and neck cancer elective surgery. Is it enough?}

\begin{abstract}
Introduction: Head and neck cancer patients are at elevated risk of SARS-CoV-2 infection; surgery in them involves risk for patients, surgeons, health personnel, medical institutions and society, since it is associated with prolonged and inadvertent production of aerosols and emergency procedures that facilitate the breach of protective measures by health personnel. Objective: To find out if pulmonary tomographic findings are sufficient to preoperatively identify patients with COVID-19. Methods: Retrospective, cross-sectional, analytical study of patients with cervical-facial neoplasms who were candidates for surgery, preoperatively evaluated by simple chest computed tomography based on the CO-RADS classification. In CO-RADS $\geq 3$ patients, surgery was suspended and PCR was performed using nasopharyngeal swab. Results: 322 patients were included, all without COVID-19 symptoms. Tomography was positive in 35 (10.87\%); in 30, nasopharyngeal swab was performed: 28 were negative and two were positive; none developed COVID-19 symptoms. Conclusions: Chest tomography is not useful as the only preoperative screening procedure for COVID-19, since its findings are nonspecific, with a high rate of false-positive results. Clinical evaluation, with PCR and tomography, is the best form of preoperative screening.
\end{abstract}

KEYWORDS: Head and neck. Cancer. COVID-19. Screening. PCR. Tomography.

Correspondencia:

*José F. Gallegos-Hernández

E-mail: gal61@ prodigy.net.mx

0016-3813/@ 2021 Academia Nacional de Medicina de México, A.C. Publicado por Permanyer. Este es un artículo open access bajo la licencia CC BY-NC-ND (http://creativecommons.org/licenses/by-nc-nd/4.0/).

Fecha de recepción: 04-07-2021

Fecha de aceptación: 05-08-2021 


\section{Introducción}

La pandemia causada por el nuevo tipo de coronavirus SARS-CoV-2 y la propagación mundial de los síntomas respiratorios agudos graves y su neumonía asociada, denominada COVID-19, han derivado en una tasa de complicaciones pulmonares posoperatorias de $51.2 \% \%^{-1} 44.4 \%$ de los pacientes requiere apoyo en unidad de cuidados intensivos y la mortalidad reportada es de $38 \%{ }^{2}$

Los pacientes oncológicos son un grupo vulnerable a infección por SARS-COV-2: su riesgo de presentar infección grave es 3.6 veces mayor ${ }^{3-6}$ y la tasa de letalidad es del doble de la población en general no oncológica (5.6\% versus $2.3 \%)$.

La tasa de complicaciones posoperatorias en pacientes COVID es mayor, incluso si se encuentran asintomáticos, por lo que es menester identificarlos para evitar riesgos. Para ello se han creado diversos protocolos para el tamizaje prequirúrgico durante la pandemia de COVID-19, para implementar un abordaje diagnóstico oportuno y certero. ${ }^{8-12}$

Normalmente, los pacientes con COVID-19 grave presentan síntomas inespecíficos como fiebre, tos y disnea; sin embargo, hay otros que pueden no manifestar síntomas y permanecer de esa forma. ${ }^{13,14}$ Esos pacientes pueden ser altamente contagiosos, por lo que se ha planteado que el cribado diagnóstico para COVID-19 en pacientes candidatos a un procedimiento terapéutico debe incluir hisopado nasofaríngeo con determinación de transcriptasa inversa en tiempo real (RT-PCR); sin embargo, los resultados pueden no tenerse en forma inmediata o bien no disponerse de la prueba.

La tomografía axial computarizada (TAC) simple de tórax es fácil de realizar y puede ayudar al diagnóstico rápido; no obstante, los resultados han sido heterogéneos y la sensibilidad reportada es de 68 a $98 \%$, con especificidad de 25 a $53 \% .^{15-19}$

En nuestro medio, durante la pandemia mundial por COVID-19, sobre todo en el primer año, con base en las recomendaciones internacionales y ante la falta rutinaria de pruebas $P C R$, efectuamos la pesquisa de infección de SARS-CoV-2 únicamente con TAC simple de tórax en pacientes asintomáticos candidatos a cirugía cervicofacial, 24 horas antes de la hora programada de la cirugía. El objetivo del presente estudio fue evaluar los resultados obtenidos con ese recurso diagnóstico y conocer si es suficiente como método de pesquisa.

\section{Métodos}

Entre el 7 de mayo de 2020 y el 7 de enero de 2021, todos los pacientes adultos programados para cirugía oncológica de cabeza y cuello en nuestra institución se sometieron a un cribado preoperatorio de COVID19 mediante TAC de tórax simple, 24 horas antes de la hora programada para su cirugía.

Antes del ingreso, a los pacientes se les aplicó un cuestionario rápido de síntomas generales para COVID-19. Quienes no se sometieron a la TAC de cribado fueron excluidos del análisis. Se realizó un estudio retrospectivo, transversal y analítico.

Las características basales, los hallazgos de la TAC según lo informado por el radiólogo y los resultados perioperatorios se extrajeron de los expedientes electrónicos.

En todas las TAC realizadas se utilizó un formato de informe estandarizado, basado en la puntuación CO-RADS, creada por la Sociedad Radiológica Alemana, ${ }^{20}$ que categoriza cada exploración en cinco grados:

- CO-RADS 1, sospecha muy baja.

- CO-RADS 2, sospecha baja.

- CO-RADS 3, resultado indeterminado.

- CO-RADS 4, sospecha alta.

- CO-RADS 5, sospecha muy alta.

La puntuación CO-RADS se basa en la conformidad de los hallazgos de la exploración con los hallazgos radiológicos típicos observados en COVID-19. A los pacientes con CO-RADS 3, 4 y 5 se les suspendió la cirugía y se les realizó prueba RT-PCR por hisopado nasofaríngeo para determinación de SARS-CoV-2. Con base en el resultado de esta prueba y una nueva TAC después de cuarentena, se decidió la reprogramación quirúrgica. Los pacientes reprogramados debían contar con al menos una prueba RT-PCR negativa y nueva TAC de tórax CO-RADS 102.

Se determinó la proporción de pacientes con una prueba de RT-PCR positiva, la aparición de síntomas relacionados con COVID-19 posterior a la TAC, así como el lapso para realizar una nueva TAC y la resolución quirúrgica del tumor primario. En el análisis estadístico, las variables continuas se presentaron como media \pm desviación estándar o mediana. Se determinaron hallazgos verdaderos positivos (VP) si se apreciaron imágenes que sugirieran COVID-19 en la TAC y el resultado de la RT-PCR fue positivo. Se calificó como hallazgos falsos positivos (FP) si en la TAC había datos de COVID-19 y la RT-PCR era negativa. 
Tabla 1. Distribución de las características generales de 35 pacientes con datos sospechosos de COVID-19 conforme TAC prehospitalaria

\begin{tabular}{|l|c|c|}
\hline Característica & \multicolumn{2}{|l|}{} \\
\hline Edad (años) & \multicolumn{2}{|c|}{$68 \pm 14$} \\
\hline & $n$ & $\%$ \\
\hline Sexo & & \\
\hline Femenino & 14 & 40 \\
\hline Masculino & 21 & 60 \\
\hline Comorbilidades & & \\
\hline Hipertensión arterial & & \\
\hline Tabaquismo & 21 & 60 \\
\hline Diabetes mellitus & 18 & 51.42 \\
\hline Dislipidemia & 17 & 48.51 \\
\hline Hipotiroidismo & 8 & 22.85 \\
\hline Tumor primario & 6 & 17.14 \\
\hline Piel no melanoma & & \\
\hline Tiroides & 17 & 48.51 \\
\hline Melanoma & 8 & 22.85 \\
\hline Cavidad oral & 6 & 17.14 \\
\hline Laringe & 3 & 8.57 \\
\hline Hallazgos tomográficos & 1 & 2.85 \\
\hline Imagen en "vidrio despulido" & & \\
\hline Engrosamientos intersticiales & 35 & 100 \\
\hline Atelectasias & 28 & 80 \\
\hline Bronquiectasias & 21 & 60 \\
\hline Consolidaciones & 16 & 45.71 \\
\hline Otros & 8 & 22.85 \\
\hline & 7 & 20 \\
\hline
\end{tabular}

\section{Resultados}

Durante el periodo de estudio se incluyeron 322 pacientes candidatos a cirugía electiva del área cervicofacial; a todos se les realizo TAC preoperatoria, $287(89.13 \%)$ fueron normales.

En 35 pacientes (10.87\%), la TAC fue positiva para datos sospechosos de COVID-19 (CO-RADS 30 mayor); las características generales de estos pacientes se muestran en la Tabla 1, entre ellas el mayor porcentaje de hombres y la hipertensión arterial como comorbilidad asociada más frecuente.

El cáncer de piel no melanoma del área de cabeza y cuello fue el padecimiento oncológico mas frecuente seguido del cáncer de tiroides y el melanoma.

En 21 pacientes, la TAC fue CO-RADS 4 o 5 y en 14, CO-RADS 3 . En 30 pacientes se tuvo acceso a la prueba de hisopado nasofaríngeo en los siguientes siete días después de la TAC, 28 tuvieron prueba negativa (falsos positivos) y dos, positiva (verdaderos positivos) a SARS-CoV-2.
Un diagrama de flujo del estudio se muestra en la Figura 1. Todos los pacientes estaban asintomáticos al momento de la TAC y ninguno desarrolló síntomas en el periodo de seguimiento.

Para la reprogramación quirúrgica se tomó una nueva TAC de tórax a 30 pacientes, con un tiempo promedio de $30.1 \pm 17.2$ días; la condición para autorizar la cirugía fue tener una puntación 102 de CO-RADS, así como haber sido negativo en el prueba de RT-PCR. Todos los pacientes permanecieron asintomáticos, incluso hasta el momento de la reprogramación de la cirugía.

Los dos casos con RT-PCR positivo no desarrollaron síntomas y tuvieron una nueva TAC negativa para la enfermedad y un nueva prueba RT-PCR negativa, por lo que finalmente se realizó la cirugía. La Figura 2 muestra la evolución de uno de los casos.

En dos pacientes se documentó progresión de la enfermedad con irresecabilidad del tumor, por lo que se les ofreció radioterapia; los restantes 28 pacientes se sometieron a cirugía, sin complicaciones respiratorias posteriores, incluso aquellos con antecedente de prueba RT-PCR positiva.

\section{Discusión}

Debido al potencial aerozolizante de la cirugía cervicofacial, diversas guías han recomendado que debe suspenderse y limitarse a los casos urgentes. ${ }^{20,21}$ Para evitar el retraso en el tratamiento oncológico se ha propuesto que el tamizaje de los casos asintomáticos y positivos a SARS-CoV-2 sea efectuado con RT-PCR y TAC pulmonar 24 horas antes de la cirugía. $^{22}$

La TAC pulmonar ha sido sugerida como un método rápido, eficaz y seguro que permite identificar cambios tempranos por infección de SARS-CoV-2, aun en pacientes asintomáticos, con lo que se evitan procedimientos de riesgo, sin necesidad de esperar varios días el resultado de la PCR. Sin embargo, su sensibilidad y especificidad son cuestionadas, porque se ha reportado un número elevado de casos falsos positivos, sobre todo durante la pandemia. Por otro lado, el diagnóstico definitivo de infección por SARSCoV-2 se lleva a cabo con la RT-PCR por hisopado nasofaríngeo. Se cree que es muy específico, pero se ha informado que su sensibilidad es baja, de 60 a $70 \% .^{8,9}$ Los falsos negativos presentan serios problemas clínicos y pueden ser necesarias varias pruebas 
Pacientes consecutivos programados para cirugía de cabeza y cuello a quienes se realizó TAC preoperatoria

Pacientes con TAC con hallazgos relacionados con COVID-19

Resultado de TAC

Resultado de RT-PCR

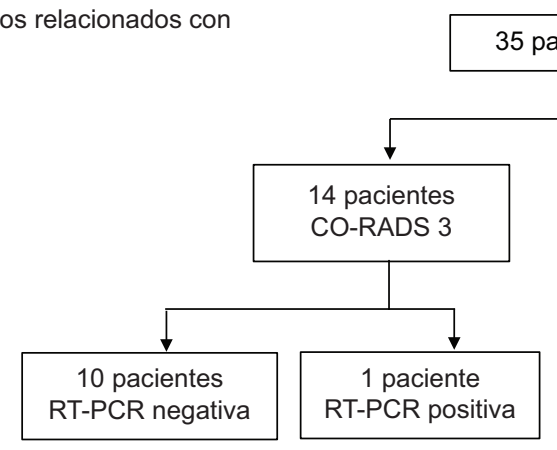

322 pacientes 287 pacientes con CO-RADS 1 o 2

5 pacientes

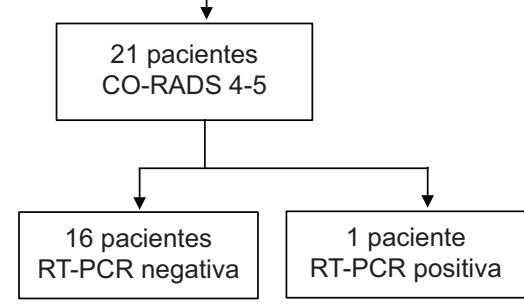

Figura 1. Diagrama de resultados de TAC y RT-PCR en pacientes con sospecha de COVID-19.

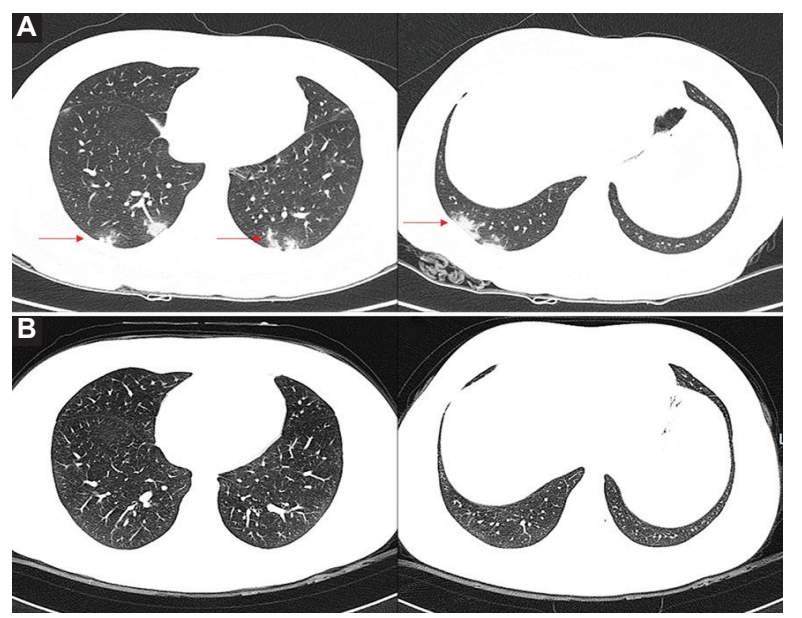

Figura 2. TAC preoperatoria de mujer de 33 años asintomática con cáncer de tiroides. A: hallazgos de imagen evaluados como CO-RADS-5 en junio de 2020. B: hallazgos de imagen evaluados como CO-RADS-1, 42 días después.

negativas en un solo caso para tener confianza en la exclusión de la enfermedad.

La evaluación clínica con pruebas de hisopado e imagen probablemente sea la mejor forma de pesquisa preoperatoria; la evidencia emergente sugiere que la sola TAC de tórax preoperatoria no contribuye a la detección de COVID-19 en los sujetos asintomáticos, aislados y probados, por lo que no se recomienda para el cribado en cirugía electiva de cáncer. ${ }^{23,24}$

En la presente serie, los hallazgos tomográficos de la neumonía por COVID-19 fueron inespecíficos y similares a los de otras infecciones pulmonares, lo que se deduce por la evolución clínica de los pacientes en quienes se suspendió la cirugía. Los hallazgos tomográficos deben correlacionarse con la evaluación clínica y los datos de laboratorio; el diagnóstico se confirma con RT-PCR. La TAC pulmonar simple por sí sola no contribuye al diagnóstico de infección por COVID-19 en sujetos asintomáticos, por lo que no se recomienda para el cribado preoperatorio en cáncer de cabeza y cuello.

\section{Conclusión}

El uso aislado de TAC pulmonar simple como tamizaje preoperatorio para COVID-19 en pacientes con cáncer de cabeza y cuello es poco útil, por el alto índice de resultados falsos-positivos. La RT-PCR, en combinación con las evaluaciones clínica y tomográfica, es probablemente el mejor método de tamizaje preoperatorio, como ha sido reportado por otros autores. . $^{25,26}$

\section{Agradecimientos}

Los autores agradecen a Hilda Miranda Bravo, enfermera especialista y terapeuta de linfedema, por su apoyo, entusiasmo y profesionalismo, y quien fuera víctima de esta terrible pandemia.

\section{Financiamiento}

La presente investigación no recibió ninguna beca específica de agencias de los sectores público, comercial o sin ánimo de lucro. 


\section{Conflicto de intereses}

Los autores declaran no tener conflicto de intereses.

\section{Responsabilidades éticas}

Protección de personas y animales. Los autores declaran que para esta investigación no realizaron experimentos en seres humanos ni en animales.

Confidencialidad de los datos. Los autores declaran que siguieron los protocolos de su centro de trabajo sobre la publicación de datos de pacientes.

Derecho a la privacidad y consentimiento informado. Los autores declaran que en este artículo no aparecen datos de pacientes.

\section{Bibliografía}

1. Nepogodiev D, Bhangu A, Glasbey JC, Li E, Omar MO, Simoes JFF, et al. Mortality and pulmonary complications in patients undergoing surgery with perioperative SARS-CoV-2 infection: an international cohort study. Lancet. 2020;396:27-38.

2. Lei S, Jiang F, Su W, Chen Ch, Chen J, Mei W, et al. Clinical characteristics and outcomes of patients undergoing surgeries during the incubation period of COVID-19 infection. EClinicalMedicine. 2020;21:100331.

3. Tian J, Yuan X, Xiao J, Zhong Q, Yang C, Liu B, et al. Clinical characteristics and risk factors associated with COVID-19 disease severity in patients with cancer in Wuhan, China: a multicentre, retrospective, cohort study. Lancet Oncol. 2020;21:893-903.

4. Yang $\mathrm{K}$, Sheng $\mathrm{Y}$, Huang $\mathrm{C}$, Jin $\mathrm{Y}$, Xiong N, Jiang $\mathrm{K}$, et al. Clinical characteristics, outcomes, and risk factors for mortality in patients with cancer and COVID-19 in Hubei, China: a multicentre, retrospective, cohort study. Lancet Oncol. 2020;21:904-913.

5. Zhang L, Zhu F, Xie L, Wang C, Wang J, Chen R, et al. Clinical characteristics of COVID-19-infected cancer patients: a retrospective case study in three hospitals within Wuhan, China. Ann Oncol. 2020;31:894-901.

6. Day AT, Sher DJ, Lee RC, Truelson JM, Myers LL, Sumer BD, et al. Head and neck oncology during COVID-19 pandemic: Reconsidering traditional treatments paradigms in light of new surgical and other multilevel risks. Oral Oncol. 2020;105:1044684.

7. Wu Z, McGoogan JM. Characteristics of and important lessons from the coronavirus disease 2019 (COVID-19) outbreak in China: summary of a report of 72314 cases from the Chinese Center for Disease Control and Prevention. JAMA. 2020;323:1239-1242.

8. Brindle ME, Doherty G, Lillemoe K, Gawande A. Approaching surgical triage during the COVID-19 pandemic. Ann Surg. 2020;272:e40-e42.

9. Argenziano M, Fischkoff K, Smith CR. Surgery scheduling in a crisis. New Engl J Med. 2020;382:e87.
10. Kowalski LP, Sanabria A, Ridge JA, Ng WT, de Bree R, Rinaldo A, et al. COVID-19 pandemic: effects and evidence-based recommendations for otolaryngology and head and neck surgery practice. Head Neck. 2020;42:1259-1267.

11. Mehanna H, Hardman JC, Shenson JA, Abou-Foul AK, Topf MC, AIFalasi $\mathrm{M}$, et al. Recomendations for head and neck surgical oncology practice in a setting of acute severe resoruce constraint during the COVID-19 pandemic: an interntional consensus. Lancet Oncol. 2020;21:e350-e359

12. Givi B, Schiff BA, Chinn SB, Clayburgh D, Iyer NG, Jalisi S, et al. Safety recommendations for evaluation and surgery of the head and neck during the COVID- 19 pandemic. JAMA Otolaryngol Head Neck Surg. 2020;146:579-584

13. Wang D, Hu B, Hu C, Zhu F, Liu X, Zhang J et al. Clinical Characteristics of 138 hospitalized patients with 2019 novel coronavirus-infected pneumonia in Wuhan, China. JAMA. 2020;323:1061-1069

14. Cheng X, Liu J, Li N, Nisenbaum E, Sun Q, Chen B, et al. Otolaryngology providers must be alert for patients with mild and asymptomatic COVID-19. Otolaryngol Head Neck Surg. 2020;162:809-810.

15. Shang $Y, X u$ C, Jiang F, Huang R, Li Y, Zhou Y, et al. Clinical characteristics and changes of chest CT features in 307 patients with common COVID-19 pneumonia infected SARS-CoV-2: a multicenter study in Jiangsu, China. Int J Infect Dis. 2020;96:157-162.

16. Homsi M, Chung M, Bernheim A, Jacobi A, King MJ, Lewis S, et al. Review of chest CT manifestations of COVID-19 infection. Eur J Radiol. 2020;7:100239.

17. Fang $Y$, Zhang $H$, Xie J, Lin $M$, Ying $L$, Pang $P$, et al. Sensitivity of chest CT for COVID19: comparison to RT-PCR. Radiology. 2020;296:E115-E117.

18. Callaway M, Harden S, Ramsden W, Beavon M, Drinkwater K, Vanburen $\mathrm{T}$, et al. A national UK audit for diagnostic accuracy of preoperative CT chest in emergency and elective surgery during COVID-19 pandemic. Clin Radiol. 2020;75:705-708.

19. Ai T, Yang Z, Hou H, Zhan Ch, Chen Ch, Lv W, et al. Correlation of chest CT and RT-PCR testing in coronavirus disease 2019 (COVID-19) in China: a report of 1014 cases. Radiology. 2020;296:E32-E40.

20. Prokop $M$, van Everdingen $W$, van Rees Vellinga $T$, van Ufford $J Q$, Stöger L, Beenien L. CO-RADS-A categorical CT assessment scheme for patients with suspected COVID-19: definition and evaluation. Radiology. 2020;27:201473.

21. Fujioka T, Takahashi M, Mori M, Tsuchiya J, Yamaga E, Horii T, et al. Evaluation of the usefulness of CO- RADS for chest CT in patients suspected of having COVID-19. Diagnostics (Basel). 2020;10:608.

22. Topf MC, Shenson JA, Holsinger FC, Wald SH, Cianfichi LJ, Rosenthal El, et al. Framework for prioritizing head and neck surgery during the COVID-19 pandemic. Head Neck. 2020;42:1159-1167.

23. Bann DV, Patel VA, Saadi R, Gniady JP, Goyal N, McGinn JD, et al. Impact of coronavirus (COVID-19) on otolaryngologic surgery: brief commentary. Head Neck. 2020;42:1227-1234.

24. Coimbra R, Edwards S, Kurihara H, Bass GA, Balogh ZJ, Tilsed J, et al. European society of trauma and emergency surgery (ESTES) recommendations for trauma and emergency surgery preparation during times of COVID-19 infection. Eur J Trauma Emerg Surg. 2020;46:505-510.

25. Givi B, Schiff BA, Chinn SB, Clayburgh D, lyer NG, Jalisi S, et al. Safety recommendations for evaluation and surgery of the head and neck during the COVID-19 pandemic. JAMA Otolaryngol Head Neck Surg. 2020;146:579-584.

26. Chetan MR, Tsakok MT, Shaw R, Xie C, Watson RA, Wing L, et al. Chest CT screening for COVID-19 in elective and emergency surgical patients: experience from a UK tertiary centre. Clin Radiol. 2020;75:599-605. 